\title{
The isomorphism relation on countable torsion free abelian groups
}

\author{
by \\ Greg Hjorth (Los Angeles, CA)
}

\begin{abstract}
The isomorphism relation on countable torsion free abelian groups is nonBorel.
\end{abstract}

1. Introduction. This note makes a comment on the isomorphism relation of countable torsion free abelian groups of infinite rank. We show that any Borel isomorphism relation on a class of countable structures may be embedded into the isomorphism relation of countable torsion free abelian groups.

In [3] a stronger result was announced, to the effect that we may actually embed the isomorphism relation of an arbitrary class of countable structures. A proof was there promised to be given in [5].

That proof, alas, was mistaken. This note instead gives a weaker result, which as it turns out is now irrelevant to the main direction of [5].

Before going further into the details we need to recall a basic concept for this area:

Definition. Let $E$ and $F$ be Borel equivalence relations on Polish spaces $X$ and $Y$. We write

$$
E \leq_{B} F
$$

if there is a Borel function $\theta: X \rightarrow Y$ such that for all $x_{1}, x_{2} \in X$,

$$
x_{1} E x_{2} \Leftrightarrow \theta\left(x_{1}\right) F \theta\left(x_{2}\right) .
$$

For $\mathcal{L}$ a countable language we can form, as described in [7], the Polish space $X_{\mathcal{L}}$ of $\mathcal{L}$ structures with underlying set $\mathbb{N}$. For $\mathcal{L}$ consisting of a single binary operation, + , the torsion free abelian groups form a closed invariant subset $\mathrm{TFA} \subset X_{\mathcal{L}}$.

Our main result is:

2000 Mathematics Subject Classification: Primary 03E15. 
TheOREM 1.1. For $\mathcal{L}^{\prime}$ a countable language and $C \subset X_{\mathcal{L}^{\prime}}$ a Borel set such that the isomorphism relation on $C,\left.\cong\right|_{C}$, is Borel, we always have

$$
\left.\cong\right|_{C} \leq\left._{B} \cong\right|_{\mathrm{TFA}}
$$

In particular this implies that $\left.\cong\right|_{\text {TFA }}$ is non-Borel, answering a question raised in [2]. However we leave open the question which the authors of [2] correctly identify as most fundamental:

Question. Let $\mathcal{L}^{\prime}$ be a countable language. Do we necessarily have

$$
\left.\cong\right|_{\mathcal{L}^{\prime}} \leq\left._{B} \cong\right|_{\mathrm{TFA}} ?
$$

In this present paper I will largely skip over giving a detailed introduction or providing extensive motivation. I feel that such an introduction has already been given in [7] and [6]. Between them, these papers should also provide the technical background and an explanation of notation not adequately defined below.

2. Group eplags. This section is devoted to a painful and detailed development of a new class of infinite rank torsion free abelian groups. The central technical lemma appears at 2.5, giving an analysis of when a given element from one of these group eplags is infinitely divisible by a given prime. This technical lemma enables the section after to embed countable iterations of the power set of $\mathbb{N}$ into torsion free abelian groups considered up to isomorphism.

Although 2.5 and the coding techniques of $\S 3$ defy easy summary, it might be helpful to make a couple of brief remarks about the philosophy of the proof. This can be most easily described by considering the case of just embedding $\mathcal{P}_{\aleph_{0}}(\mathcal{P}(\mathbb{N}))$ into the isomorphism relation on torsion free abelian groups.

A way in which we might effect such an encoding is to first enumerate infinitely many distinct primes, $\left(p_{n}\right)_{n \in \mathbb{N}}$. Then given $\left\{a_{n}: n \in \mathbb{N}\right\} \subset \mathcal{P}(\mathbb{N})$ we can describe a subgroup of $\mathbb{Q}^{\mathbb{N}}$, where the element $\delta_{n}$ (the function taking value 1 at $n$ and zero elsewhere) is infinitely divisible by a prime $p$ if and only if $p=p_{i}$ for some $i$ with $i \in a_{n}$. With suitable care this can be done so that the isomorphism type of the corresponding group only depends on the set $\left\{a_{n}: n \in \mathbb{N}\right\}$ and so that for each non-zero $g$ in the group, the set of primes infinitely dividing $g$ will either be empty or equal to $\left\{p_{i}: i \in a_{n}\right\}$, for some $n \in \mathbb{N}$.

The case of $\mathcal{P}_{\aleph_{0}}(\mathcal{P}(\mathbb{N}))$ is misleadingly simple, because at this level every object being coded is a subset of some predetermined countable set. In the general case we need to be able to build up sets of sets, and sets of sets of sets, and so on, without any previously described limitations. The complications required for this further coding are reflected in 2.5 and 3.1 below. 
Definition. A graph is a pair $(V, E)$ where $V$ is a set and $E$ is a subset of the unordered pairs $\{\{v, w\}: v, w \in V, v \neq w\}$ of elements in $V$.

Notation. $\mathbb{P}$ denotes the set of primes.

Definition. A prime labeled graph is a triple $(V, E, f)$ where $(V, E)$ is a graph and $f: V \cup E \rightarrow \mathbb{P}$. It is said to be excellent if

(i) $f[V] \cap f[E]=\emptyset$; that is to say, vertices and edges are always assigned distinct primes;

(ii) for any $p \in \mathbb{P},\left(V, f^{-1}[\{p\}]\right)$ contains no cycles; in other words, if we restrict our attention to edges labeled by a single prime then the resulting graph is a union of disjoint trees.

I will use the acronym eplag for excellent prime labeled graph.

Definition. Given an eplag $(V, E, f)$ we define the corresponding group eplag, denoted by $\mathcal{G}(V, E, f)$. We first consider the group consisting of all formal sums

$$
\sum_{v \in V_{0}} q_{v} \cdot v
$$

where $V_{0}$ is a finite subset of $V$ and $\left(q_{v}\right)_{v \in V_{0}}$ is a corresponding array of non-zero rationals; we add elements in this group in the obvious way, and thus it becomes a torsion free abelian group. We then let $\mathcal{G}(V, E, f)$ be the abelian group consisting of all elements that may be represented in the form

$$
g=\sum_{v \in V_{0}} \frac{k_{v} \cdot v}{f(v)^{l_{v}}}+\sum_{\{v, w\} \in E_{0}} \frac{n_{\{v, w\}} \cdot(v+w)}{f(\{v, w\})^{m_{\{v, w\}}}},
$$

where $V_{0}$ is a finite subset of $V, E_{0}$ is a finite subset of $E$, with each $k_{v}, n_{\{v, w\}} \in \mathbb{Z}$ and $l_{v}, m_{\{v, w\}} \in \mathbb{N}$.

In other words, $\mathcal{G}(V, E, f)$ can be thought of as isomorphic to a subgroup of the direct sum

$$
\bigoplus_{V} \mathbb{Q}
$$

with the product group structure, where we insist that each $\chi_{\{v\}}$ (the characteristic function of $\{v\}$, taking $\chi_{\{v\}}(w)=1$ when $w=v$, and assuming value 0 otherwise) is divisible by all powers of $f(v)$ while each $\chi_{\{v\}}+\chi_{\{w\}}$ is divisible by all powers of $f(\{v, w\})$ for $\{v, w\} \in E$. In particular every element of $\mathcal{G}(V, E, f)$ can be written in the form

$$
\sum_{v \in V_{1}} q_{v} \cdot v
$$

for some finite $V_{1} \subset V$ and associated collection $\left\{q_{v}: v \in V_{1}\right\}$ of rationals. 
Definition. Let $(V, E, f)$ be an eplag and $\varrho: V \rightarrow\{-1,0,1\}$ a function. We define a resulting homomorphism $\varphi_{\varrho}: \mathcal{G}(V, E, f) \rightarrow \mathbb{Q}$ by

$$
\varphi_{\varrho}\left(\sum_{v \in V_{0}} q_{v} \cdot v\right)=\sum_{v \in V_{0}} q_{v} \varrho(v) .
$$

Definition. Let $\mathcal{G}(V, E, f)$ be a group eplag and let $p$ be a prime. A homomorphism $\psi: \mathcal{G}(V, E, f) \rightarrow \mathbb{Q}$ is $p$-mindful if $\psi=\varphi_{\varrho}$ as above for some $\varrho: V \rightarrow\{-1,0,1\}$ with

$$
\varrho\left(w_{0}\right)=-\varrho\left(w_{1}\right) \quad \text { for all }\left\{w_{0}, w_{1}\right\} \in E \cap f^{-1}[\{p\}] .
$$

LEMMA 2.1. If $C \subset V$ is a connected component of $\left(V, f^{-1}[\{p\}]\right)$ containing some given vertex $v$ and $\varphi_{0}, \varphi_{1}: \mathcal{G}(V, E, f) \rightarrow \mathbb{Q}$ are $p$-mindful homomorphisms with $\varphi_{0}(v), \varphi_{1}(v)$ both non-zero, then for any $g \in \mathcal{G}(V, E, f)$ of the form

$$
g=\sum_{v \in C_{0}} q_{v} \cdot v
$$

with $C_{0}$ a finite subset of $C$, we have

$$
\varphi_{0}(g)=0 \text { if and only if } \varphi_{1}(g)=0 .
$$

Proof. The point is that there are only two possible two-colorings of $\left(C, f^{-1}[\{p\}]\right)$ by $\{-1,1\}$, and hence only two possible non-trivial $p$-mindful homomorphisms for the subgroup of $\mathcal{G}(V, E, f)$ generated by $\{w: w \in C\}$. Each of these homomorphisms is the mirror image of the other, in the sense that $\varphi_{0}(g)=-\varphi_{1}(g)$ for all $g$ in their common domain.

LEMMA 2.2. Suppose $C_{1}, \ldots, C_{k}$ are distinct connected components of $\left(V, f^{-1}[\{p\}] \cap E\right)$ and for each $i \leq k$ we have

$$
g_{i}=\sum_{v \in V_{i}} r_{v} \cdot v
$$

for some $V_{i} \subset C_{i}$. Suppose $g=g_{1}+\ldots+g_{k}$. Then the following statements are equivalent:

(I) $\varphi(g)=0$ for each $p$-mindful $\varphi$.

(II) $\varphi\left(g_{i}\right)=0$ for each $i$ and each $p$-mindful $\varphi$.

Proof. This follows since if $\mathcal{F}$ enumerates the components of $\left(V, f^{-1}[\{p\}]\right.$ $\cap E)$, then any $p$-mindful $\varphi$ may be written as a sum $\varphi: g \mapsto \sum_{C \in \mathcal{F}} \varphi_{C}(g)$ where each $\varphi_{C}$ is $p$-mindful and assumes the value 0 outside of $C$.

Lemma 2.3. Let $\mathcal{G}(V, E, f)$ be a group eplag, $p \in \mathbb{P}, v \in V$. Then there is a function $\varrho: V \rightarrow\{-1,0,1\}$ such that

(i) $\varrho(w) \neq 0$ if and only if there are $v_{0}, v_{1}, \ldots, v_{k} \in V$ with each $\left\{v_{i}, v_{i+1}\right\}$ $\in f^{-1}[\{p\}] \cap E$, and $v_{0}=v, v_{k}=w$; in other words, @ takes non-zero values exactly on the component of $\left(V, f^{-1}[\{p\}] \cap E\right)$ containing $v$; 
(ii) for $\left\{w_{0}, w_{1}\right\} \in f^{-1}[\{p\}] \cap E$ we have

$$
\varrho\left(w_{0}\right)=-\varrho\left(w_{1}\right)
$$

in other words, @ provides a two-coloring of the component on which it is non-zero.

Proof. This is essentially a restatement of the well known fact that every tree admits a two-coloring.

Lemma 2.4. Let $(V, E, f)$ be an eplag. Suppose $p \in \mathbb{P}$ and $\left(T, f^{-1}[\{p\}] \cap\right.$ $\{\{v, w\}: v, w \in T\})$ is a finite subtree of $\left(V, f^{-1}[\{p\}] \cap E\right)$ and $g \in$ $\mathcal{G}(V, E, f)$ has the form

$$
g=\sum_{v \in T} r_{v} \cdot v
$$

for rationals $\left\{r_{v}: v \in T\right\}$. Suppose $\varphi(g)=0$ for every $p$-mindful homomorphism $\varphi: \mathcal{G}(V, E, f) \rightarrow \mathbb{Q}$. Then $g$ is divisible by every power of $p$.

Proof. We prove the lemma by induction on the cardinality $|T|$ of $T$. If this is 1 , then $g$ would have to be the zero element of the group, since for $g=q \cdot v$ we can always define a $p$-mindful homomorphism $\varphi$ which assumes a value $\varphi(v)=1$; and certainly zero is divisible by all powers of $p$.

So now suppose

$$
g=\sum_{v \in T} r_{v} \cdot v
$$

$|T|=n+1 \geq 2$, and that the lemma has been proved for any trees $T^{\prime}$ with $\left|T^{\prime}\right| \leq n$. Then we may choose a node $v_{0} \in T$ that is terminal; let $v_{1}$ be the unique vertex in $T$ with $\left\{v_{0}, v_{1}\right\} \in f^{-1}[\{p\}]$. We can let

$$
h=r_{v_{0}} \cdot v_{0}+r_{v_{0}} \cdot v_{1}
$$

it follows from the definition of $\mathcal{G}(V, E, f)$ that $h$ is divisible by all powers of $p$; and it follows from the definition of mindfulness that $\varphi(h)=0$ for every $p$-mindful homomorphism $\varphi$. Thus by assumption on $g$,

$$
\varphi(g-h)=0
$$

for every $p$-mindful homomorphism $\varphi: \mathcal{G}(V, E, f) \rightarrow \mathbb{Q}$, and so by inductive assumption $g-h$ is divisible by every power of $p$. But then $g$ must also be divisible by every power of $p$.

In the proof of the following proposition we use without explicit mention this consequence of unique factorization: If $p, p_{0}, p_{1}, \ldots, p_{k}$ are non-zero primes, $n, n_{0}, \ldots, n_{k}$ are non-zero integers, with $n$ not divisible by $p$, and $m, m_{0}, \ldots, m_{k}$ are positive integers and

then $p_{i}=p$ for some $i \leq k$.

$$
\frac{n}{p^{m}}=\sum_{i \leq k} \frac{n_{i}}{p_{i}^{m_{i}}},
$$


Proposition 2.5. Let $\mathcal{G}(V, E, f)$ be a group eplag, $p \in \mathbb{P}, V_{0} \subset V$ finite, and $g \in \mathcal{G}(V, E, f)$ of the form

$$
g=\sum_{v \in V_{0}} q_{v} \cdot v
$$

with each $q_{v} \neq 0$. Then $g$ is divisible by all powers of $p$ in $\mathcal{G}(V, E, f)$ if and only if either:

(i) $V_{0} \subset V \cap f^{-1}[\{p\}]$, or

(ii) $\varphi(g)=0$ for each p-mindful homomorphism $\varphi: \mathcal{G}(V, E, f) \rightarrow \mathbb{Q}$.

Proof. For notational simplicity let us assume each $q_{v} \in \mathbb{Z}$. We can make this simplifying assumption since the euclidean algorithm shows that $g$ is divisible by every power of $p$ if and only if every integer multiple of $g$ is divisible by every power of $p$.

$(\Leftarrow)$ : First suppose (i) holds. Then it is immediate from the definitions that for each $v \in V_{0}$ and $n \in \mathbb{N}$ we have $p^{-n} \cdot v \in \mathcal{G}(V, E, f)$, and hence $g$ is indeed divisible by every power of $p$.

So instead suppose (ii) holds. Note that we can find finitely many components $W_{1}, \ldots, W_{k}$ of $\left(V, E \cap f^{-1}[\{p\}]\right)$ such that

$$
g=g_{1}+\ldots+g_{k}
$$

where each $g_{i}$ is a rational linear combination of $\left\{v: v \in W_{i}\right\}$. Then $g$ will be divisible by all powers of $p$ if each $g_{i}$ is divisible by each power of $p$. Thus by 2.2 we may as well assume that $k=1$; that is to say, $g$ is contained in a single component of $\left(V, E \cap f^{-1}[\{p\}]\right)$.

With this in hand, we can write

$$
g=\sum_{v \in T} l_{v} \cdot v
$$

where $T \subset V$ is finite and $\left(T, f^{-1}[\{p\}] \cap\{\{v, w\}: v, w \in T\}\right)$ forms a tree. And then we are done by appealing to 2.4 .

$(\Rightarrow)$ : Assume for a contradiction that (i) and (ii) both fail.

Claim (1). There is $h \in G$ such that $\varphi(h)=0$ for every $p$-mindful homomorphism $\varphi: \mathcal{G}(V, E, f) \rightarrow \mathbb{Q}$ and such that if

$$
h+g=\sum_{v \in W} r_{v} \cdot v
$$

is a representation of $g+h$ with each $r_{v} \neq 0$ for $v \in W$, then for each connected component $C$ of $\left(V, f^{-1}[\{p\}]\right)$ there is at most one $v \in W \cap C$.

Proof of claim. For each connected component $C$ of $\left(V, f^{-1}[\{p\}] \cap E\right)$ that meets $V_{0}$ choose some single $v_{C} \in C$ and a two-coloring $\varrho_{C}: C \rightarrow\{-1,1\}$ of the tree

$$
\left(C, f^{-1}[\{p\}] \cap\left\{\left\{w_{1}, w_{2}\right\} \in E: w_{1}, w_{2} \in C\right\}\right)
$$


with $\varrho_{C}\left(v_{C}\right)=1$. We then let

$$
h_{C}=\sum_{v \in C \cap V_{0}}-q_{v} \cdot v+\sum_{v \in C \cap V_{0}} q_{v} \varrho_{C}(v) \cdot v_{C} .
$$

By 2.1 we find that $\varphi\left(h_{C}\right)=0$ for any $p$-mindful homomorphism $\varphi$. Thus if we let $\mathcal{F}$ enumerate the connected components of $\left(V, f^{-1}[\{p\}] \cap E\right)$ meeting $V_{0}$, then $h=\sum_{C \in \mathcal{F}} h_{C}$ is as required. Claim

Thus by the already proved direction $\Leftarrow$ of the proposition we see that $h$ is divisible by every power of $p$. And hence so is $g+h$. Moreover $g+h$ fails (ii) since $g$ fails (ii). In particular, $g+h \neq 0$.

Hence, after possibly replacing $g$ by $g+h$ we may make the simplifying assumption that for each connected component $C$ of $\left(V, f^{-1}[\{p\}] \cap E\right)$ there is at most a single vertex $w_{C}$ with $w_{C} \in V_{0} \cap C$ in the representation of $g$. Thus for some finite collection $\mathcal{H}$ of connected components of $\left(V, f^{-1}[\{p\}]\right)$ and some finite collection $\left(a_{C}\right)_{C \in \mathcal{H}}$ of integers we have

$$
g=\sum_{C \in \mathcal{H}} a_{C} \cdot w_{C}
$$

Choose some sufficiently large $n$ that no $a_{C}$ is divisible by $p^{n}$-and thus the coefficient of $a_{C}$ appearing in $g / p^{n}$ must have a power of $p$ in its denominator. Now $g$ is divisible by $p^{n}$ in $\mathcal{G}(V, E, f)$, and thus we may write

$$
\frac{g}{p^{n}}=\sum_{v \in V_{1}} \frac{k_{v} \cdot v}{f(v)^{l_{v}}}+\sum_{\{v, w\} \in E_{1}} \frac{n_{\{v, w\}} \cdot(v+w)}{f(\{v, w\})^{m_{\{v, w\}}}}
$$

for finite $V_{1} \subset V, E_{1} \subset E$, each $m_{\{v, w\}}>0$, each $n_{\{v, w\}}$ indivisible by $f(\{v, w\})$. For each $C \in \mathcal{H}$ let $T_{C} \subset C$ be the smallest possible subtree of $\left(V, f^{-1}[\{p\}]\right)$ such that if $\left\{v_{0}, v_{1}\right\} \in E_{1}$ with $v_{0}, v_{1} \in C$ then $v_{0}, v_{1} \in T_{C}$.

Claim (2). $f^{-1}[\{p\}] \cap V=\emptyset$.

Proof of claim. Inspecting the definition of $\mathcal{G}(V, E, f)$ we see that every $h$ in the group may be written in the form

$$
h=\sum_{v \in W_{1}} \frac{b_{v} \cdot v}{\left(f(v) f\left(\left\{w_{0, v}, v\right\}\right) f\left(\left\{w_{1, v}, v\right\}\right) \ldots f\left(\left\{w_{k(v), v}, v\right\}\right)\right)^{c_{v}}}
$$

with each $\left\{w_{i, v}, v\right\} \in E$, each $b_{v} \in \mathbb{Z}$, and each $c_{v} \in \mathbb{N}$. In particular then the assumption of $g$ 's unlimited divisibility by $p$ along with our hypothesis that some $v \in V_{0}$ has $f(v) \neq p$ implies that there is some $w$ with

$$
\{w, v\} \in E, \quad f(\{w, v\})=p .
$$

Then by the definition of excellent prime labeled graph we must have $f^{-1}[\{p\}] \cap V=\emptyset$. $\mathbf{m}_{\text {Claim }}$

Claim (3). For no $C \in \mathcal{H}$ do we have $T_{C}=\emptyset$; that is to say, for every $C \in \mathcal{H}$ there is some $v_{0}, v_{1} \in C$ with $\left\{v_{0}, v_{1}\right\} \in E_{1}$. 
Proof of claim. Since for each $C \in \mathcal{H}$ we may write

$$
\frac{g}{p^{n}}=\frac{a_{C} \cdot w_{C}}{p^{n}}+\sum_{v \in V_{0}, v \neq w_{C}} \frac{q_{v} \cdot v}{p^{n}}
$$

and

$$
\frac{g}{p^{n}}=\sum_{v \in V_{1}} \frac{k_{v} \cdot v}{f(v)^{l_{v}}}+\sum_{\{v, w\} \in E_{1}} \frac{n_{\{v, w\}} \cdot(v+w)}{f(\{v, w\})^{m_{\{v, w\}}}},
$$

and since each $a_{C}$ is indivisible by $p^{n}$, we see that for any $C \in \mathcal{H}$ there is some $v$ with

$$
\left\{v, w_{C}\right\} \in E_{1}, \quad f\left(\left\{v, w_{C}\right\}\right)=p . \mathbf{m}_{\text {Claim }}
$$

So let us choose $C_{0} \in \mathcal{H}$ and $v_{0} \in T_{C_{0}}$ with $v_{0} \neq w_{C_{0}}$ but $v_{0}$ terminal in $T_{C_{0}}$ - that is to say, there is exactly one other $v_{1} \in T_{C_{0}}$ with $\left\{v_{0}, v_{1}\right\} \in E$. This is possible since $T_{C_{0}}$ is a finite tree and since, by the above claim, it has at least two elements. Referring back to the representation

$$
\frac{g}{p^{n}}=\sum_{C \in \mathcal{H}} \frac{a_{C} \cdot w_{C}}{p^{n}}
$$

and using the fact that $v_{0} \neq w_{C}$ for all $C \in \mathcal{H}$ we see that

$$
\frac{k_{v_{0}} \cdot v_{0}}{f\left(v_{0}\right)^{l_{v_{0}}}}+\sum_{\left\{v_{0}, w\right\} \in E_{1}} \frac{n_{\left\{v_{0}, w\right\}} \cdot\left(v_{0}+w\right)}{f\left(\left\{v_{0}, w\right\}\right)^{m_{\left\{v_{0}, w\right\}}}}
$$

can be expressed as a linear combination of $\left\{w \in V: w \neq v_{0}\right\}$, and thus

$$
\frac{k_{v_{0}}}{f\left(v_{0}\right)^{l_{v_{0}}}}+\sum_{\left\{v_{0}, w\right\} \in E_{1}} \frac{n_{\left\{v_{0}, w\right\}}}{f\left(\left\{v_{0}, w\right\}\right)^{m_{\left\{v_{0}, w\right\}}}}=0 .
$$

But now the assumption on $v_{0}$ gives us a single $w_{0}$ with

$$
\left\{v_{0}, w_{0}\right\} \in E_{1}, \quad f\left(\left\{v_{0}, w_{0}\right\}\right)=p .
$$

Thus we obtain primes $p_{1}, \ldots, p_{N}$ distinct from $p$ with

$$
\frac{k_{v_{0}}}{f\left(v_{0}\right)^{l_{v_{0}}}}+\frac{n_{\left\{w_{0}, v_{0}\right\}}}{p^{m_{\left\{w_{0}, v_{0}\right\}}}}+\sum_{i \leq N} \frac{n_{i}}{p_{i}^{m_{i}}}=0 .
$$

Since $f\left(v_{0}\right) \neq p$ we have at last reached a contradiction.

\section{Coding hereditarily countable sets}

Definition. Following [7], we define $\mathcal{P}^{\alpha}(\mathbb{N})$ by induction on $\alpha<\omega_{1}$. We begin with $\mathcal{P}^{0}(\mathbb{N})=\mathbb{N}$ and then inductively define $\mathcal{P}^{\alpha}(\mathbb{N})$ for $\alpha>0$ to be $\bigcup_{\beta<\alpha} \mathcal{P}^{\beta}(\mathbb{N})$ along with the set of all countable subsets of $\bigcup_{\beta<\alpha} \mathcal{P}^{\beta}(\mathbb{N})$. For $A \in \bigcup_{\alpha<\omega_{1}} \mathcal{P}^{\alpha}(\mathbb{N})$ we let $\operatorname{Rk}(A)$ be the least $\alpha$ such that $A \in \mathcal{P}^{\alpha}(\mathbb{N})$.

We also define $\operatorname{TC}(A)$ in the following way. If $A=m \in \mathcal{P}^{0}(\mathbb{N})$ we define $\mathrm{TC}(A)$ to be the set $\{n: n<m\}$. We then continue inductively, so that 
when $A \in \mathcal{P}^{\alpha}(\mathbb{N})$ for some $\alpha>0$ then

$$
\mathrm{TC}(A)=A \cup \bigcup_{B \in A} \mathrm{TC}(B) .
$$

Notation. For the rest of this section fix a countable ordinal $\alpha$ and a collection of distinct primes

$$
\left\{p_{\beta}: \beta \leq \alpha, \beta \neq 0\right\} \cup\left\{q_{n}: n \in \mathbb{N}\right\} \cup\left\{\widehat{q}_{\gamma, \beta}: \gamma<\beta \leq \alpha\right\} \cup\left\{\bar{p}_{\gamma, \beta}: \gamma<\beta \leq \alpha\right\} .
$$

The goal of this section is to show that given this ordinal $\alpha$ and this collection of primes we can canonically associate to each $A \in \mathcal{P}^{\alpha}(\mathbb{N})$ a corresponding torsion free abelian group $\mathcal{H}_{A}$ such that

$$
A_{0}=A_{1} \quad \text { if and only if } \quad \mathcal{H}_{A_{0}} \cong \mathcal{H}_{A_{1}} \text {. }
$$

In this section we simply concentrate on describing the construction. It is only in the following two sections that any significance can be attached to the endeavor - the next section observes this particular construction to be suitably "Borel", and the section after notes consequences for the isomorphism relation on torsion free abelian groups. This present section should really be thought of as one long definition.

We first build a group $\mathcal{G}_{A}$, for each $A \in \mathcal{P}^{\alpha+1}(\mathbb{N})$, and verify that

$$
\mathrm{TC}\left(A_{1}\right) \neq \mathrm{TC}\left(A_{2}\right) \quad \text { implies } \quad \mathcal{G}_{A_{1}} \nsucceq \mathcal{G}_{A_{2}} .
$$

We can then define $\mathcal{H}_{A}=\mathcal{G}_{\{A\} \cup \operatorname{TC}(A)}$ for $A \in \mathcal{P}^{\alpha}(\mathbb{N})$ and check that indeed $A_{1}=A_{2} \Leftrightarrow \mathcal{H}_{A_{1}} \cong \mathcal{H}_{A_{2}}$.

Definition. For $A \in \mathcal{P}^{\alpha+1}(\mathbb{N})$ we let $V_{A}$ consist of all $\left\langle A_{1}, \ldots, A_{n}\right\rangle$ where

(i) $n \in \mathbb{N}$;

(ii) each $A_{i} \in \mathrm{TC}(A)$;

(iii) $\operatorname{Rk}\left(x_{i+1}\right)<\operatorname{Rk}\left(x_{i}\right)$ for each $i<n$.

We let $E_{A}$ consist of all pairs

$$
\left\{\left\langle A_{1}, \ldots, A_{n}\right\rangle,\left\langle A_{1}, \ldots, A_{n}, A_{n+1}\right\rangle\right\},
$$

where $\left\langle A_{1}, \ldots, A_{n}, A_{n+1}\right\rangle \in V_{A}$. We define a prime labeling $f_{A}: V_{A} \cup E_{A} \rightarrow \mathbb{P}$ as follows:

$$
\begin{gathered}
f\left(\left\langle A_{1}, \ldots, A_{n}\right\rangle\right)= \begin{cases}q_{m} & \text { if } \operatorname{Rk}\left(A_{n}\right)=0 \text { and } A_{n} \text { is the natural number } m ; \\
p_{\beta} & \text { if } \operatorname{Rk}\left(A_{n}\right)=\beta>0 ;\end{cases} \\
f\left(\left\{\left\langle A_{1}, \ldots, A_{n}\right\rangle,\left\langle A_{1}, \ldots, A_{n}, A_{n+1}\right\rangle\right\}\right) \\
= \begin{cases}\widehat{q}_{\gamma, \beta} & \text { if } \operatorname{Rk}\left(A_{n}\right)=\beta, \operatorname{Rk}\left(A_{n+1}\right)=\gamma, \text { and } A_{n+1} \in A_{n} ; \\
\bar{p}_{\gamma, \beta} & \text { if } \operatorname{Rk}\left(A_{n}\right)=\beta, \operatorname{Rk}\left(A_{n+1}\right)=\gamma, \text { and } A_{n+1} \notin A_{n} .\end{cases}
\end{gathered}
$$

Thus $\left(V_{A}, E_{A}, f_{A}\right)$ is an eplag for any $A \in \mathcal{P}^{\alpha+1}(\mathbb{N})$; and from this eplag we can pass to the corresponding group. 
Definition. For any $A \in \mathcal{P}^{\alpha+1}(\mathbb{N})$ we let

$$
\mathcal{G}_{A}=\mathcal{G}\left(V_{A}, E_{A}, f_{A}\right),
$$

as defined in the last section.

Definition. For any $A \in \mathcal{P}^{\alpha+1}(\mathbb{N})$ and $\beta<\alpha$ we define the notions of $\beta$-good and a corresponding $\beta$ th evaluation function $\pi_{\beta}$ by a simultaneous induction.

We say that $g \in \mathcal{G}_{A}$ is 0 -good if for some $m \in \mathbb{N}, g$ is divisible in $\mathcal{G}_{A}$ by all powers of $q_{m}$; we then let

$$
\pi_{0}(g)=m ;
$$

note that by construction there is at most one such $m$, and hence $\pi_{0}(g)$ is well defined for $g$-good. For $\beta>0$ we say that $g$ is $\beta$-good if it is divisible by all powers of $p_{\beta}$ and for all $\gamma<\beta$ and all $\gamma$-good $h$ we have either

(a) there is a $\gamma$-good $h^{\prime}$ with $\pi_{\gamma}(h)=\pi_{\gamma}\left(h^{\prime}\right)$ and $g+h^{\prime}$ divisible by all powers of $\widehat{q}_{\gamma, \beta}$, or

(b) there is a $\gamma$-good $h^{\prime}$ with $\pi_{\gamma}(h)=\pi_{\gamma}\left(h^{\prime}\right)$ and $g+h^{\prime}$ divisible by all powers of $\bar{p}_{\gamma, \beta}$.

We then let $\pi_{\beta}(g)$ be the set of all $\pi_{\gamma}(h)$ such that $\gamma<\beta, h$ is $\gamma$-good, and $g+h$ is divisible by all powers of $\widehat{q}_{\gamma, \beta}$.

Literally of course all these definitions should be made with explicit reference to $A$. We should define $\beta$-good in $\mathcal{G}_{A}$ and we should write something like $\pi_{\mathcal{G}_{A}, \gamma}$ to show the dependence of the evaluation map on the group $\mathcal{G}_{A}$.

In practice the intention will be clear.

Lemma 3.1. (a) Suppose

$$
g=\sum_{i \leq k} r_{i} \cdot v_{i} \in \mathcal{G}_{A}
$$

is $\beta$-good with each $r_{i} \in \mathbb{Q}, r_{i} \neq 0, v_{i} \neq v_{j}$ for $i \neq j$, and $v_{i}=\left\langle A_{1}^{i}, \ldots, A_{l(i)}^{i}\right\rangle$. Then for all $i, j \leq k$,

$$
A_{l(i)}^{i}=A_{l(j)}^{j}, \quad \operatorname{Rk}\left(A_{l(i)}^{i}\right)=\beta,
$$

and for $A^{*}$ the common value of the $A_{l(i)}^{i}$ we have

$$
\pi_{\beta}(g)=A^{*} \text {. }
$$
and

(b) If $\left\langle A_{1}, \ldots, A_{l}\right\rangle \in V_{A}$ with $\operatorname{Rk}\left(A_{l}\right)=\beta$, then $\left\langle A_{1}, \ldots, A_{l}\right\rangle$ is $\beta$-good

$$
\pi_{\beta}\left(\left\langle A_{1}, \ldots, A_{l}\right\rangle\right)=A_{l} .
$$

Proof. We prove these by simultaneous induction. The base case $\beta=0$ follows quickly from the definitions and 2.5. So assume that $\beta>0$ and that the lemma is established for all $\gamma<\beta$. 
First suppose that $g=\sum_{i \leq k} r_{i} \cdot v_{i} \in \mathcal{G}_{A}$ is $\beta$-good, each $r_{i}$ a non-zero rational, each $v_{i}=\left\langle A_{1}^{i}, \ldots, A_{l(i)}^{i}\right\rangle$, with $v_{i} \neq v_{j}$ for $i \neq j$, and assume for a contradiction that $\widehat{A} \in A_{l(0)}^{0} \backslash A_{l(1)}^{1}$. It follows from the definition of goodness that each $\left\langle A_{0}^{i}, \ldots, A_{l(i)}^{i}\right\rangle$ is divisible by all powers of $p_{\beta}$, and hence, by 2.5 , at each $i$ we have $\operatorname{Rk}\left(A_{l(i)}^{i}\right)=\beta$.

Thus $\widehat{A}$ must have $\operatorname{Rk}(\widehat{A})=\gamma$ for some $\gamma<\beta$. And then by the inductive assumption $\langle\widehat{A}\rangle$ is $\gamma$-good with $\pi_{\gamma}(\langle\widehat{A}\rangle)=\widehat{A}$. Then by the definition of $\beta$ goodness of $g$ there is some $\gamma$-good $h$ with $\pi_{\gamma}(h)=\widehat{A}$ and $g+h$ either divisible by every power of $\widehat{q}_{\gamma, \beta}$ or divisible by every power of $\bar{p}_{\gamma, \beta}$. Assume for simplicity that it is divisible by every power of $\widehat{q}_{\gamma, \beta}$; the other case is similar. We can write

$$
h=\sum_{i \leq k^{\prime}} s_{i} \cdot w_{i}
$$

with each $w_{i} \in V_{A}, s_{i}$ a non-zero rational, $w_{i} \neq w_{j}$ for $i \neq j$. Then by the inductive assumption applied to the $\gamma$-goodness of $h$ each $w_{i}$ is of the form

$$
w_{i}=\left\langle B_{1}^{i}, \ldots, B_{l^{\prime}(i)}^{i}, \widehat{A}\right\rangle .
$$

At this point we can define $\varrho: V_{A} \rightarrow\{-1,0,1\}$ by

$$
\begin{aligned}
\varrho\left(\left\langle A_{0}^{1}, \ldots, A_{l(1)}^{1}\right\rangle\right) & =1, & & \\
\varrho\left(\left\langle A_{0}^{1}, \ldots, A_{l(1)}^{1}, B\right\rangle\right) & =-1 & & \text { for any } B \in A_{l(1)}^{1}, \\
\varrho(v) & =0 & & \text { for all other } v \in V_{A} .
\end{aligned}
$$

Then $\varphi_{\varrho}$ defined by

$$
\sum q_{v} \cdot v \mapsto \sum q_{v} \varrho(v)
$$

provides a $\widehat{q}_{\gamma, \beta}$-mindful homomorphism which assumes the value $\varphi_{\varrho}(g+h)=$ $r_{1} \neq 0$, contradicting Proposition 2.5's criteria for infinite $\widehat{q}_{\gamma, \beta}$ divisibility.

To complete the inductive step we also need to note that (b) holds for any $\left\langle A_{1}, \ldots, A_{l}\right\rangle \in V_{A}$ with $\operatorname{Rk}\left(A_{l}\right)=\beta$. This follows routinely from the definition of $\beta$-good and the inductive assumption.

Corollary 3.2. $\mathrm{TC}\left(A_{1}\right) \neq \mathrm{TC}\left(A_{2}\right)$ implies $\mathcal{G}_{A_{1}} \nRightarrow \mathcal{G}_{A_{2}}$.

Proof. This follows since

$$
\begin{aligned}
& \left\{\pi_{\beta}(g): g \in \mathcal{G}_{A_{1}}, g \text { is } \beta \text {-good, } \beta \leq \alpha\right\}=\mathrm{TC}\left(A_{1}\right), \\
& \left\{\pi_{\beta}(g): g \in \mathcal{G}_{A_{2}}, g \text { is } \beta \text {-good, } \beta \leq \alpha\right\}=\mathrm{TC}\left(A_{2}\right),
\end{aligned}
$$

and for any group $G$ the set $\left\{\pi_{\beta}(g): g \in G, g\right.$ is $\beta$-good, $\left.\beta \leq \alpha\right\}$ is an invariant of the isomorphism type of $G$.

Now for any $A \in \mathcal{P}^{\alpha}(\mathbb{N})$ we can define

$$
\mathcal{H}_{A}=\mathcal{G}_{\{A\} \cup \operatorname{TC}(A)}
$$


to obtain an assignment

$$
\mathcal{P}^{\alpha}(\mathbb{N}) \hookrightarrow \mathrm{TFA} \cong, \quad A \mapsto \mathcal{H}_{A},
$$

which injects $\mathcal{P}^{\alpha}(\mathbb{N})$ into the countable torsion free abelian groups considered up to isomorphism.

But of course the simple existence of such an injection amounts to nothing more than $\left|\mathcal{P}^{\alpha}(\mathbb{N})\right| \leq 2^{\aleph_{0}}$. The important point is that this injection is, in the sense made precise shortly, reasonably definable.

4. Coding. We recall a method of coding elements of $\mathcal{P}^{\alpha}(\mathbb{N})$ by equivalence classes in a Polish space.

Definition. Following [7] we let $\mathcal{L}(\alpha)$ be the language generated by unary relations $\left(R_{\beta}\right)_{\beta \leq \alpha}$, binary relations $\epsilon, E, F$, and constant symbols $v_{0},\left(r_{n}\right)_{n \in \mathbb{N}}$. We let $X_{\mathcal{L}(\alpha)}$ be the Polish space of $\mathcal{L}(\alpha)$ structures equipped with the usual topology generated by quantifier free formulas. We then let $P^{\alpha}$ be the subspace consisting of the structures

$$
\mathcal{M}=\left\langle\mathbb{N} ; r_{0}, r_{1}, \ldots, v_{0}, \epsilon, E, F, R_{0}, R_{1}, \ldots, R_{\beta}, \ldots, R_{\alpha}\right\rangle,
$$

where:

(i) $\mathbb{N}$ is partitioned by the relations $\left(R_{\beta}\right)_{\beta \leq \alpha}$; the constant terms $\left(r_{n}\right)_{n \in \mathbb{N}}$ enumerate $R_{0}$ without repetitions; $R_{\alpha}=\left\{v_{0}\right\}$;

(ii) $E$ is an irreflexive symmetric relation on $\mathbb{N} \backslash R_{0}$;

(iii) $E$ provides a tree structure on $\mathbb{N} \backslash R_{0}$ with root $v_{0}$; this tree structure has the property that if we define $x<^{*} y$ if there is one-to-one $f: k+2 \rightarrow$ $\mathbb{N} \backslash R_{0}$ with $f(0)=v_{0}, f(k)=y, f(k+1)=x$, then the partial order $<^{*}$ is wellfounded in the sense of having no infinite descending chain, and $R_{\gamma}(x)$ holds exactly when $x$ has rank $\gamma$ in this tree; in particular, $v_{0}$ has rank $\alpha$;

(iv) $F \subset R_{0} \times R_{1}$.

With this all in place we can assign an evaluation $\|x\|$ to each $x \in \mathbb{N}$, with

$$
\begin{array}{ll}
\|x\|=n & \text { if } x \in R_{0} \text { and } x=r_{n}, \text { while } \\
\|x\|=\left\{n \in \mathbb{N}: F\left(r_{n}, x\right)\right\} & \text { for } x \in R_{1}
\end{array}
$$

(and hence $x$ terminal in the tree structure on $\mathbb{N} \backslash R_{0}$ ); and

$$
\|x\|=\left\{\|y\|: \text { the shortest path from } v_{0} \text { to } y \text { passes through } x\right\} .
$$

(v) We require that $\epsilon$ satisfy

$$
x \epsilon y \Leftrightarrow\|x\| \in\|y\| .
$$

With all this in place we can finally define

$$
\|\mathcal{M}\|=\left\|v_{0}\right\| \text {. }
$$


Some remarks.

First of all this definition suffers from the same formal defect as some earlier definitions. We should literally be writing $r_{0}^{\mathcal{M}}, r_{1}^{\mathcal{M}}, \ldots, E^{\mathcal{M}}, \ldots$ and so on to distinguish the relation symbols from the relations which arise in their interpretation in $\mathcal{M}$. And again we should write $\|x\|^{\mathcal{M}}$ to indicate the dependence of the evaluation on the structure $\mathcal{M}$.

Hopefully the reader will forgive this ellipsis as well as all the earlier defects.

The second remark is to give some motivation. The idea is that we want to represent elements of $\mathcal{P}^{\alpha}(\mathbb{N})$ by countable trees. We use the $E$ relation to describe this tree structure. The nodes of the trees correspond to the various sets appearing in the transitive closure of some $A \in \mathcal{P}^{\alpha}(\mathbb{N})$, and some representative of $B_{1}$ appears below a representative of $B_{2}$ if and only if $B_{1} \in B_{2}$; we deliberately allow that a given $B \in \mathrm{TC}(A)$ may reappear in many different branches of the tree. We give a special status to the natural numbers; they comprise $R_{0}$ and are individually named by the $r_{n}$ 's. The higher $R_{\gamma}$ 's layer $\mathcal{M}$ into sediments based on the rank of the represented sets.

The $\epsilon$ relation comes along for free, simply recapitulating the relation $\|y\| \in\|x\|$. I leave it only because it appeared in [7].

Following 1.2-1.5 of [7] we conclude that $P^{\alpha}$ is a Borel subset of $X_{\mathcal{L}(\alpha)}$, and hence a standard Borel set in its own right, and that

$$
\left\{\|\mathcal{M}\|: \mathcal{M} \in P^{\alpha}\right\}=\mathcal{P}^{\alpha}(\mathbb{N}),
$$

as well as that the set of $(x, y, \mathcal{M}) \in \mathbb{N} \times \mathbb{N} \times P^{\alpha}$ such that $\|x\|=\|y\|$ (calculated from the point of view of $\mathcal{M}$ ) is Borel as a subset of $\mathbb{N} \times \mathbb{N} \times X_{\mathcal{L}(\alpha)}$. If we let $\cong_{\alpha}$ be the isomorphism relation on elements of $P^{\alpha}$ then for all $\mathcal{M}, \mathcal{N} \in P^{\alpha}$ we have

$$
\mathcal{M} \cong \mathcal{N} \Leftrightarrow\|\mathcal{M}\|=\|\mathcal{N}\|
$$

Definition. For each $\mathcal{M} \in P^{\alpha}$ let $S_{0}(\mathcal{M})$ be the set of $x \in \mathbb{N}$ such that for all smaller natural numbers $y \in \mathbb{N}$ we have $\|x\| \neq\|y\|$.

Thus $(\|x\|)_{x \in S_{0}(\mathcal{M})}$ enumerates without repetitions the set $\{\|\mathcal{M}\|\} \cup$ $\mathrm{TC}(\|\mathcal{M}\|)$. From the above remarks we see that $\left\{(x, \mathcal{M}) \in \mathbb{N} \times P^{\alpha}\right.$ : $\left.x \in S_{0}(\mathcal{M})\right\}$ is Borel as a subset of $\mathbb{N} \times X_{\mathcal{L}(\alpha)}$.

Definition. Let $\pi: \mathbb{N}<\mathbb{N} \hookrightarrow \mathbb{N}$ be the usual Gödel pairing function, providing a bijection between $\mathbb{N}<\mathbb{N}$ and $\mathbb{N}$. Then for $\mathcal{M} \in P^{\alpha}$ we let $S_{1}(\mathcal{M})=\left\{\pi\left(x_{1}, \ldots, x_{n}\right):\right.$

each $x_{i} \in S_{0}(\mathcal{M})$, and $\operatorname{Rk}\left(\left\|x_{i}\right\|\right)>\operatorname{Rk}\left(\left\|x_{i+1}\right\|\right)$ for $\left.i<n\right\}$.

Again the set $\left\{(a, \mathcal{M}) \in \mathbb{N} \times P^{\alpha}: a \in S_{1}(\mathcal{M})\right\}$ is Borel as are

$\left\{\left(\pi\left(x_{1}, \ldots, x_{n}\right), \pi\left(x_{1}, \ldots, x_{n}, x_{n+1}\right), \mathcal{M}\right) \in N \times \mathbb{N} \times \mathbb{P}^{\alpha}:\right.$

$$
\left.\pi\left(x_{1}, \ldots, x_{n}\right), \pi\left(x_{1}, \ldots, x_{n}, x_{n+1}\right) \in \mathcal{M},\left\|x_{n+1}\right\| \in\left\|x_{n}\right\|\right\}
$$


and

$$
\begin{aligned}
& \left\{\left(\pi\left(x_{1}, \ldots, x_{n}\right), \pi\left(x_{1}, \ldots, x_{n}, x_{n+1}\right), \mathcal{M}\right) \in \mathbb{N} \times \mathbb{N} \times \mathbb{P}^{\alpha}:\right. \\
& \left.\pi\left(x_{1}, \ldots, x_{n}\right), \pi\left(x_{1}, \ldots, x_{n}, x_{n+1}\right) \in \mathcal{M},\left\|x_{n+1}\right\| \notin\left\|x_{n}\right\|\right\} .
\end{aligned}
$$

And clearly from the definition of $P^{\alpha}$ we see that for each $\beta \leq \alpha$ the set

$$
\left\{\left(\pi\left(x_{1}, \ldots, x_{n}\right), \mathcal{M}\right) \in \mathbb{N} \times P^{\alpha}: \pi\left(x_{1}, \ldots, x_{n}\right) \in S_{1}(\mathcal{M}), \operatorname{Rk}\left(\left\|x_{n}\right\|\right)=\beta\right\}
$$

is Borel for any $\beta \leq \alpha$.

And thus all the sets of the form

$\left\{(p, a, \mathcal{M}): a \in S_{1}(\mathcal{M}), a=\pi\left(x_{1}, \ldots, x_{n}\right)\right.$ for some $n, x_{1}, \ldots, x_{n}$ with $\operatorname{Rk}\left(\left\|x_{n}\right\|\right)=\beta$ for some $\left.p_{\beta}=p\right\}$,

$\left\{(p, a, \mathcal{M}): a \in S_{1}(\mathcal{M}), a=\pi\left(x_{1}, \ldots, x_{n}\right)\right.$ for some $n, x_{1}, \ldots, x_{n}$ with $\left.\operatorname{Rk}\left(\left\|x_{n}\right\|\right)=0,\left\|x_{n}\right\|=m, q_{m}=p\right\}$,

$\left\{(p, a, b, \mathcal{M}): a, b \in S_{1}(\mathcal{M}), a=\pi\left(x_{1}, \ldots, x_{n}\right), b=\pi\left(x_{1}, \ldots, x_{n}, x_{n+1}\right)\right.$

for some $n, x_{1}, \ldots, x_{n}, x_{n+1}$ with $\operatorname{Rk}\left(\left\|x_{n}\right\|\right)=\beta, \operatorname{Rk}\left(\left\|x_{n+1}\right\|\right)=\gamma$,

and $\left.\left\|x_{n+1}\right\| \in\left\|x_{n}\right\|, \widehat{q}_{\gamma, \beta}=p\right\}$,

and

$$
\begin{array}{r}
\left\{(p, a, b, \mathcal{M}): a, b \in S_{1}(\mathcal{M}), a=\pi\left(x_{1}, \ldots, x_{n}\right), b=\pi\left(x_{1}, \ldots, x_{n}, x_{n+1}\right)\right. \\
\text { for some } n, x_{1}, \ldots, x_{n}, x_{n+1} \text { with } \operatorname{Rk}\left(\left\|x_{n}\right\|\right)=\beta, \operatorname{Rk}\left(\left\|x_{n+1}\right\|\right)=\gamma, \\
\text { and } \left.\left\|x_{n+1}\right\| \notin\left\|x_{n}\right\|, \bar{p}_{\gamma, \beta}=p\right\},
\end{array}
$$

are Borel.

Thus it follows that the set $M$ of all sequences

$$
\left(\mathcal{M},\left(r_{1}, a_{1}\right),\left(r_{2}, a_{2}\right), \ldots,\left(r_{l}, a_{l}\right)\right) \in P^{\alpha} \times(\mathbb{Q} \times \mathbb{N})^{<\mathbb{N}}
$$

such that

(i) $\left(a_{1}, \ldots, a_{l}\right)$ is a strictly increasing sequence of integers;

(ii) each $a_{i}=\pi\left(x_{1}^{i}, \ldots, x_{N(i)}^{i}\right)$ for some $N(i), x_{1}^{i}, \ldots, x_{N(i)}^{i} \in \mathbb{N}$;

(iii) $\sum_{i \leq l} r_{i} \cdot\left\langle\left\|x_{1}^{i}\right\|, \ldots,\left\|x_{N(i)}^{i}\right\|\right\rangle \in \mathcal{G}_{\{\|\mathcal{M}\|\} \cup \mathrm{TC}(\|\mathcal{M}\|)}$

is Borel. Moreover we deduce then that the induced group structure on the fibers gives rise to a Borel subset of $M \times M \times M$, in the sense that the set of all

$$
\left(\mathcal{M},\left(a_{1}, r_{1}\right), \ldots,\left(a_{l}, r_{l}\right),\left(b_{1}, s_{1}\right), \ldots,\left(b_{k}, s_{k}\right),\left(c_{1}, t_{1}\right), \ldots,\left(c_{m}, t_{m}\right)\right)
$$

satisfying (i)-(iii) below is Borel:

(i) $\left(\mathcal{M},\left(a_{1}, r_{1}\right), \ldots,\left(a_{l}, r_{l}\right)\right),\left(\mathcal{M},\left(b_{1}, s_{1}\right), \ldots,\left(b_{k}, s_{k}\right)\right),\left(\mathcal{M},\left(c_{1}, t_{1}\right), \ldots\right.$ $\left.\ldots,\left(c_{m}, t_{m}\right)\right)$ are in $M$; 
(ii) each $a_{i}=\pi\left(x_{1}^{i}, \ldots, x_{N(i)}^{i}\right)$ for some $N(i), x_{1}^{i}, \ldots, x_{N(i)}^{i} \in \mathbb{N}$, each $b_{i}=$ $\pi\left(y_{1}^{i}, \ldots, y_{M(i)}^{i}\right)$ for some $M(i), y_{1}^{i}, \ldots, y_{M(i)}^{i} \in \mathbb{N}$, each $c_{i}=\pi\left(z_{1}^{i}, \ldots, z_{L(i)}^{i}\right)$ for some $L(i), y_{1}^{i}, \ldots, y_{L(i)}^{i} \in \mathbb{N}$;

(iii) $\sum_{i \leq l} r_{i} \cdot\left\langle\left\|x_{1}^{i}\right\|, \ldots,\left\|x_{N(i)}^{i}\right\|\right\rangle+\sum_{i \leq k} s_{i} \cdot\left\langle\left\|y_{1}^{i}\right\|, \ldots,\left\|y_{M(i)}^{i}\right\|\right\rangle$

in $\mathcal{G}_{\{\|\mathcal{M}\|\} \cup \mathrm{TC}(\|\mathcal{M}\|)}$.

$$
=\sum_{i \leq m} t_{i} \cdot\left\langle\left\|z_{1}^{i}\right\|, \ldots,\left\|z_{L(i)}^{i}\right\|\right\rangle
$$

Let $Y$ denote the space $(\mathbb{Q} \times \mathbb{N})^{<\mathbb{N}}$, in its usual Borel structure. Then $M$ is a Borel subset of $X_{\mathcal{L}(\alpha)} \times Y$ with countable sections. Thus $\left({ }^{1}\right)$ we can produce a countable sequence $\left(f_{n}\right)_{n \in \mathbb{N}}$ of Borel functions, with each $f_{n}: P^{\alpha} \rightarrow Y$, such that for any $\mathcal{M} \in P^{\alpha},\left(f_{n}(\mathcal{M})\right)_{n \in \mathbb{N}}$ enumerates without repetition the set of $y \in Y$ such that $(\mathcal{M}, y) \in M$. Thus, again using the uniformization theorem for Borel subsets of the plane with countable sections, we may find a Borel function $\theta: P^{\alpha} \rightarrow \mathbb{N}^{\mathbb{N} \times \mathbb{N}}$ such that for all $\mathcal{M} \in P^{\alpha}$ and $l, m, n \in \mathbb{N}$,

$$
f_{l}(\mathcal{M})+f_{m}(\mathcal{M})=f_{n}(\mathcal{M}) \quad \text { if and only if } \quad(\theta(\mathcal{M}))(l, m)=n ;
$$

and thus $\theta(\mathcal{M})$ provides an abelian group structure on $\mathbb{N}$ isomorphic to $\mathcal{G}_{\{\|\mathcal{M}\|\} \cup \operatorname{TC}(\|\mathcal{M}\|)}$.

\section{So what?}

Definition. Let TFA be the set of all $G: \mathbb{N} \times \mathbb{N} \rightarrow \mathbb{N}$ which provide a torsion free abelian group structure on the natural numbers. We let $\left.\cong\right|_{\text {TFA }}$ be the isomorphism relation on TFA, so that

$$
\left.G_{1} \cong\right|_{\mathrm{TFA}} G_{2}
$$

if and only if there is some permutation $\psi$ of the natural numbers such that for all $m, n \in \mathbb{N}$,

$$
\psi\left(G_{1}(m, n)\right)=G_{2}(\psi(m), \psi(n)) .
$$

If we equip $\mathbb{N}^{\mathbb{N} \times \mathbb{N}}$ with the usual product topological structure then TFA becomes a Borel set. The last section showed that for all $\alpha<\omega_{1}$,

$$
\cong_{\alpha} \leq\left._{B} \cong\right|_{\mathrm{TFA}} \text {. }
$$

Thus $\left.\cong\right|_{\text {TFA }}$ is non-Borel by

$\left({ }^{1}\right)$ See $[8,18.10]$ for a proof of this classical theorem; indeed, [8] is probably the right reference for all the descriptive set theory used here. 
THEOREM 5.1 (Harrington). There is no Borel equivalence relation E with

$$
\cong_{\alpha} \leq_{B} E
$$

all $\alpha<\omega_{1}$. (See $\left.[7,5.11]\right)$ for a proof.)

Harrington's argument can be used to show that there is no Borel $E$ with each $\cong_{\alpha}$ absolutely $\Delta_{2}^{1}$ reducible $\left({ }^{2}\right)$ to $E$, and thus we conclude, under very general notions of reducibility, that not only is $\cong_{\mathrm{TFA}}$ not Borel but it is not even reducible to any Borel equivalence relation.

It is known, as found for instance in [1], that if $C$ is a Borel class of countable structures with $\left.\cong\right|_{C}$ Borel, then there is some $\alpha<\omega_{1}$ with

$$
\left.\cong\right|_{C} \leq_{B} \cong_{\alpha}
$$

Thus we see that any such $C$ is Borel reducible to the isomorphism relation on TFA.

We can also factor in the results of Friedman and Stanley, who in [2] showed that there is no absolutely $\Delta_{2}^{1}$ assignment of elements of $2^{<\omega_{1}}$ as complete invariants for elements of $P^{2}$ up to $\cong_{2}$. Thus in particular there is no Ulm-type classification of torsion free abelian groups - a result first pointed out in [9] on the basis of very different reasoning.

And we may continue in this fashion. Similar arguments show that $\cong_{3}$ is not absolutely $\Delta_{2}^{1}$ reducible to $\mathcal{P}_{\aleph_{0}}\left(2^{<\omega_{1}}\right)$, the set of countable subsets of $2^{<\omega_{1}}$, and thus the torsion free abelian groups cannot be assigned elements of $\mathcal{P}_{\aleph_{0}}\left(2^{<\omega_{1}}\right)$ as complete invariants.

And so on.

\section{References}

[1] H. Friedman, Borel and Baire reducibility, Fund. Math. 164 (2000), 61-69.

[2] H. Friedman and L. Stanley, A Borel reducibility theory for classes of countable structures, J. Symbolic Logic 54 (1989), 899-914.

[3] G. Hjorth, Around nonclassifiability for countable torsion free abelian groups, in: Abelian Groups and Modules (Dublin, 1998), Trends Math., Birkhäuser, Basel, 1999, 269-292.

[4] -, Classification and Orbit Equivalence Relations, Math. Surveys Monogr. 75, Amer. Math. Soc., Providence, RI, 2000.

[5] —, On invariants for measure preserving transformations, Fund. Math. 169 (2000), $51-84$.

[6] G. Hjorth and A. S. Kechris, Borel equivalence relations and classifications of countable models, Ann. Pure Appl. Logic 82 (1996), 221-272.

[7] G. Hjorth, A. S. Kechris and A. Louveau, Borel equivalence relations induced by actions of the symmetric group, ibid. 92 (1998), 63-112.

[8] A. S. Kechris, Classical Descriptive Set Theory, Springer, New York, 1995.

$\left({ }^{2}\right)$ In the sense of $\S 9.1$ of $[4]$. 
[9] G. Melles, One cannot show from ZFC that there is an Ulm-type classification of the countable torsion-free abelian groups, in: Set Theory of the Continuum (Berkeley, CA, 1989), H. Judah, W. Just and W. H. Woodin (eds.), Math. Sci. Res. Inst. Publ. 26, Springer, New York, 1992, 293-309.

6363 MSB, Mathematics

UCLA

Los Angeles, CA 90095-1555, U.S.A.

E-mail: greg@math.ucla.edu

Web: www.math.ucla.edu/ greg

Received 8 January 2001;

in revised form 13 September 2002 